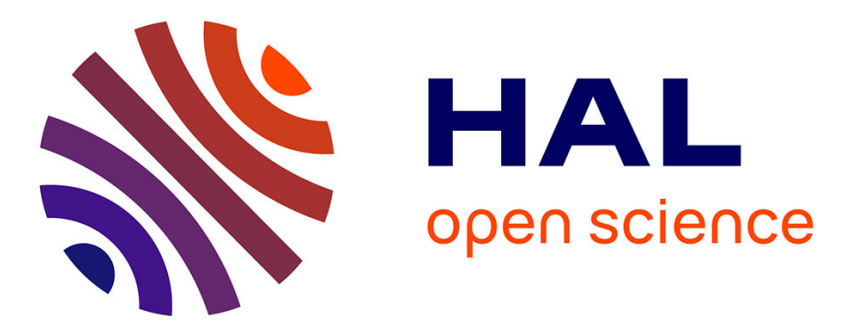

\title{
Controlling Ambipolar Transport and Voltage Inversion in Solution-Processed Thin-Film Devices through Polymer Blending
}

Tim Leydecker, Marco A. Squillaci, Fabiola Liscio, Emanuele Orgiu, Paolo Samorì

\section{To cite this version:}

Tim Leydecker, Marco A. Squillaci, Fabiola Liscio, Emanuele Orgiu, Paolo Samorì. Controlling Ambipolar Transport and Voltage Inversion in Solution-Processed Thin-Film Devices through Polymer Blending. Chemistry of Materials, In press, 10.1021/acs.chemmater.8b04819 . hal-02054650

\author{
HAL Id: hal-02054650 \\ https://hal.science/hal-02054650
}

Submitted on 1 Mar 2019

HAL is a multi-disciplinary open access archive for the deposit and dissemination of scientific research documents, whether they are published or not. The documents may come from teaching and research institutions in France or abroad, or from public or private research centers.
L'archive ouverte pluridisciplinaire HAL, est destinée au dépôt et à la diffusion de documents scientifiques de niveau recherche, publiés ou non, émanant des établissements d'enseignement et de recherche français ou étrangers, des laboratoires publics ou privés. 


\title{
Controlling ambipolar transport and voltage inversion in solution-pro- cessed thin-film devices through polymer blending
}

\author{
Tim Leydecker, ${ }^{\dagger}$ Marco A. Squillaci, ${ }^{\dagger}$ Fabiola Liscio, ${ }^{\ddagger}$ Emanuele Orgiu, ${ }^{\dagger},{ }^{*}$ and Paolo Samorì ${ }^{\dagger}{ }^{*}$ \\ †University of Strasbourg, CNRS, ISIS UMR 7006, 8 Alleé Gaspard Monge, F-67000 Strasbourg, France \\ ₹ Istituto per la Microelettronica e Microsistemi (IMM) - CNR, via Gobetti 101, 40129 Bologna, Italy
}

\begin{abstract}
Ambipolar semiconductors are attracting a great interest as building blocks for photovoltaics and logic applications. Field-effect transistors built on solution-processable ambipolar materials hold strong promise for the engineering of large-area low-cost logic circuits with a reduced number of devices components. Such devices still suffer from a number of obstacles including the challenging processing, the low Ion/Ioff, the unbalanced mobility and the low gain in CMOS-like circuits. Here, we demonstrate that the simple approach of blending commercially available $\mathrm{n}$ - and p-type polymers such as P(NDI20D-T2), P3HT, PCD-TPT, PDVT-8 and IIDDT-C3 can yield high-performing ambipolar field-effect transistors with balanced mobilities and Ion/Ioff $>10^{7}$. Each single component was studied separately and upon blending by means of electrical characterization, ambient UPS, AFM and GIWAXS to unravel the correlation between the morphology/structure of the semiconducting films and their functions. Blends of $\mathrm{n}$ - and $\mathrm{p}$-type semiconductors were used to fabricate CMOS-like inverter circuits with state-of-the-art gains over 160 in the case of P(NDI2OD-T2) blended with PDVT-8. Significantly, our blending approach was successful in producing semiconducting films with balanced mobilities for each of the 4 tested semiconductor blends although the films displayed different structural and morphological features. Our strategy, which relies on establishing a correlation between ambipolar performances, film morphology, molecular structure and blending ratio, is extremely efficient and versatile, thus it could be applied to a wide range of polymers or solution processable small molecules.
\end{abstract}

Ambipolar field-effect transistors are devices whose active layer can transport electrons and holes. ${ }^{1-3}$ Their capability to transport both types of carriers gives rise to three different operation states: a hole current on state, an off state, and the electron current on state. Such feature is required in order to fabricate logic circuits with a lower number of device components if compared to circuit architectures relying only on unipolar semiconductors. ${ }^{4}$ Ambipolar nature is observed in most organic semiconductors by careful consideration of the relative energy level alignment mismatch and the physico-chemical nature of the semiconductor/dielectric interface. In particular, hydroxyl-free gate dielectric interface has been found to considerably improve $n$ type operation in otherwise-unipolar ( $p$-type) semiconductors. ${ }^{5}$ However, the latter condition is not sufficient to observe balanced ambipolar transport, which remains a rare and most sought-after propriety in organic materials. ${ }^{6}$

In order to obtain electron and hole transport in a device, a viable approach relies on the use of ambipolar organic semiconductors. ${ }^{7-10}$ When high-performing semiconductor are exploited, hole and electron mobilities exceeding 1 $\mathrm{cm}^{2} /$ Vs can be reached. However, these ambipolar semiconductors usually exhibit a much lower $\mathrm{I}_{\text {on }} / \mathrm{I}_{\text {off }}\left(\mathrm{I}_{\text {on }} / \mathrm{I}_{\text {off }} \leq 10^{3}\right)$ than their unipolar counterpart, which is often ascribed to the higher off current ( $I_{D S}=I_{\min }$ ) measured in ambipolar films. Furthermore, $p$-type / $n$-type mobility ratio in a single ambipolar semiconductor can be hardly controlled by molecular design and processing, therefore leading to very unbalanced ambipolar transport. ${ }^{11}$
The possibility to attain ambipolar behavior in organic field-effect transistors by combining $n$-type and $p$-type semiconductors was first demonstrated by sequential thermal sublimation of semiconductor layers, ${ }^{12-13}$ and later by cosublimation of two complementary small molecules. ${ }^{14}$ The fabrication of devices with two semiconductor layers deposited from solution has also been pursued thanks to the easy processability and its applicability to polymers. Strategies include physical separation of $p$ - and $n$-channel through an intermediate titanium suboxide layer, ${ }^{15}$ the contact-filmtransfer method (transferring a polymer film on top of another polymer film) ${ }^{16}$ and the sequential deposition from orthogonal solvents. ${ }^{17-18}$ Ambipolar blends using polymers and small molecules have been recently reported, 4, 19-20 with devices featuring hole and electron mobility of 0.12 $\mathrm{cm}^{2} / \mathrm{Vs}$ and $0.04 \mathrm{~cm}^{2} / \mathrm{Vs}$, respectively ${ }^{21}$ but still lagging behind in terms of $\mathrm{I}_{\text {on }} / \mathrm{I}_{\text {off }}$ (below $10^{3}$ ). Despite these initial low performances, co-deposition of two polymers from a solution can be considered being the fastest and most straightforward approach to implement bicomponent deposition. Ambipolar polymer/polymer blends have for instance been produced, with balanced mobilities up to $3 \times 10^{-3} \mathrm{~cm}^{2} / \mathrm{Vs}$ in the case of P3HT blended with P(NDI2OD-T2.22-26 While these results point to the great potential of the blending approach, issues such as relatively low mobilities and $\mathrm{I}_{\mathrm{on}} / \mathrm{I}_{\text {off }}$ $\left(10^{3}\right)$ still need to be addressed. Furthermore, most of the literature on this topic focuses on a given ratio between the two components in the blend, thereby making it difficult to establish the correlation between ambipolar performances, film morphology, molecular structure and blending ratio. 
Here, we fabricate high-performance bi-component polymeric transistors with balanced ambipolar transport as revealed by simultaneous high electron and hole mobility, large threshold voltage window $\left(\mathrm{V}_{\mathrm{Th}, \mathrm{n}}-\mathrm{V}_{\mathrm{Th}, \mathrm{p}}\right)$ and high $\mathrm{I}_{\text {on }} / \mathrm{I}_{\text {off, }}$ being therefore ideal platforms for the development of inverter-based circuits. We explore the correlation between blending ratio and ambipolar behavior, and compare the morphology of films based on blended materials with that of single-component films. Due to the strong correlation between electron transport and order at the supramolecular level,27-28 mono-component and bi-component films are studied by means of Grazing Incidence Wide Angle X-ray Scattering. Finally, complementary-like circuits are fabricated to demonstrate the applicability of this approach to the design of logic circuits. As a proof-of-concept, the prototypical $n$-type polymer poly([N,N'-bis(2-octyldodecyl)naphthalene-1,4,5,8-bis(dicarboximide)-2,6-diyl]-alt-5,5'-

(2,2'-bithiophene)) (P(NDI2OD-T2)) was combined via blending with four different $p$-type semiconductors, i.e. poly(3-hexylthiophene-2,5-diyl) (P3HT), poly(3decyltetradecyl isoindigo-alt-dithiophene) (IIDDT-C3), poly(3,6-dithiophen-2-yl-2,5-di(2-octyldodecyl)pyr-

rolo[3,4-c]pyrrole-1,4-dione-alt-thienylenevinylene-2,5-yl) (PDVT-8) and poly[4-(4,4-dihexadecyl-4H-cyclopenta[1,2b:5,4-b']dithiophen-2-yl)-alt [1,2,5] thiadiazolo [3,4-c]pyridine] (PCD-TPT) (Figure 1).

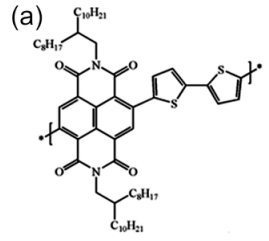

(d)

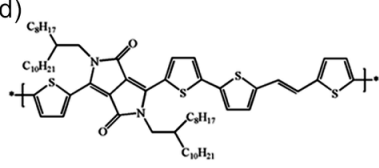

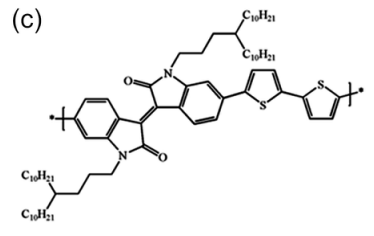

(e)

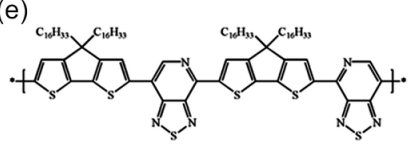

Figure 1. Molecular structures of (a) the $n$-type semiconductor P(NDI2OD-T2), and the $p$-type semiconductors (b) P3HT, (c) IIDDT-C3, (d) PDVT-8, and (e) PCD-TPT. Average molecular weights and polydispersity of the used materials are reported in Supporting Table 2.

To unravel the electrical characteristics of the individual polymeric semiconductors, transfer and output curves (Fig. $\mathbf{S 1}$ and $\mathbf{S 2}$ in the SI) were recorded in single-component bottom-contact bottom-gate transistors, produced by spincoating a $5 \mathrm{mg} / \mathrm{mL}$ polymer solution in chloroform on an octadecyltrichlorosilane (OTS) treated pre-patterned $\mathrm{SiO}_{2}$ substrate with interdigitated Au electrodes. In the case of P(NDI20D-T2) (Fig. S3), the transfer curves revealed a large $\mathrm{I}_{\mathrm{on}} / \mathrm{I}_{\text {off }}$ exceeding $10^{6}$ both in linear and saturation regime, while $n$-type mobility $\left(\mu_{\mathrm{n}}\right)$ amounted to an average of $0.018 \mathrm{~cm}^{2} / \mathrm{Vs}$ in saturation regime, in agreement with previous reports on the same polymer in bottom-gate geometry ${ }^{29}$. Conversely, its $p$-type mobility $\left(\mu_{\mathrm{p}}\right)$ was found to be very low $\left(<10^{-6} \mathrm{~cm}^{2} / \mathrm{Vs}\right)$. P3HT films which did not undergo any thermal or solvent annealing, were found to behave as a typical $p$-type semiconductor $\left(\mu_{\mathrm{p}}=0.015 \mathrm{~cm}^{2} / \mathrm{Vs}\right.$, Ion $/$ Ioff $\left.>10^{6}\right)$, while exhibiting negligible $n$-type charge transport $\left(\mu_{\mathrm{n}}<10^{-7} \mathrm{~cm}^{2} / \mathrm{Vs}\right)$ (Fig. S4a). Interestingly, when the same processing conditions used for P3HT were employed for PCD-TPT, PDVT-8 and IIDDT-C3, high $\mu_{\mathrm{p}}$ (amounting to $0.06,0.60$ and $0.08 \mathrm{~cm}^{2} / \mathrm{Vs}$, respectively) and significantly lower $\mu_{\mathrm{n}}$ (Fig. S4b-d) were observed. In particular, PDVT-8 and IIDDT-C3 featured $n$-type mobilities around 10-4 $\mathrm{cm}^{2} / \mathrm{Vs}$, making them very unbalanced yet ambipolar semiconductors. PCD-TPT was the $p$-type polymer featuring the highest $n$-type mobility $\left(5.4 \times 10^{-3} \mathrm{~cm}^{2} / \mathrm{Vs}\right)$, thus leading to a ten-fold difference in hole and electron mobility. While all these polymers exhibited good Ion/Ioff exceeding $10^{6}$ in the linear regime, $I_{\text {on }} / I_{\text {off }}$ of PCD-TPT was found to be lower in the saturation regime $\left(10^{4}\right)$.

In order to cast light onto the role of morphology on the electrical characteristics of organic polymers, ${ }^{30}$ the upper surface of the active layers' regions comprised within the transistor channel were imaged by means of Atomic Force Microscopy (AFM) (Fig. S5). The average surface rootmean-squared roughness ( $\left.\mathrm{R}_{\mathrm{RMS}}\right)$, as determined on topographical images mapping an area of $6.25 \mu \mathrm{m}^{2}$, are summarized in Supporting Table 3. P(NDI2OD-T2) films clearly exhibit the presence of oriented lamellar structures, forming a smooth film with an $\mathrm{R}_{\mathrm{RMS}}$ of $0.64 \mathrm{~nm}$, consistently with morphologies observed in literature. ${ }^{31}$ P3HT and PDVT-8 films did not exhibit any predominant morphological feature, but they displayed two different $\mathrm{R}_{\mathrm{RMS}}$ values of $0.51 \mathrm{~nm}$ and $0.91 \mathrm{~nm}$, respectively. The surface of PCD-TPT and in particular of IIDDT-C3 showed high roughness $\left(\mathrm{R}_{\mathrm{RMS}}=0.8\right.$ $\mathrm{nm}$ and $1.78 \mathrm{~nm}$, respectively), and are characterized by the presence of rigid and elongated domains featuring lamellar structure.

Each $p$-type semiconductor was individually blended with P(NDI2OD-T2) in $\mathrm{CHCl}_{3}$; once assembled in the channel of the transistor, these bi-component systems would act as the active layer in bottom-contact bottom-gate devices. Five different weight blending ratios were studied for each P(NDI20D-T2): $p$-type polymer combination, i.e. 100:0, 20:80, 50:50, 80:20 and 0:100. Field-effect mobilities for each blend ratio are reported in Fig. 2.
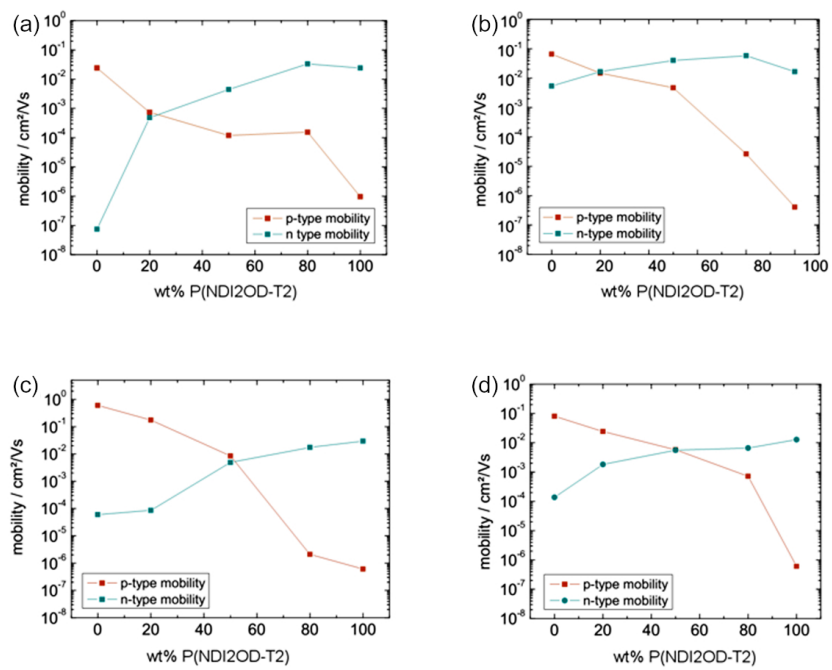

Figure 2. Electrical performances of blends with different polymer-ratios. Average saturation regime mobility (over 16 transistors, 4 at each L: $2.5 \mu \mathrm{m}, 5 \mu \mathrm{m}, 10 \mu \mathrm{m}$ and $20 \mu \mathrm{m})$ as a function of the percentage in weight of P(NDI2OD-T2) in the semiconductor solution deposited over the electrodes. 
Blends of P(NDI2OD-T2) with (a) P3HT, (b) PCD-TPT, (c) PDVT-8 and (d) IIDDT-C3. All mobility values, highest measured mobility and threshold voltages are reported in Supporting Table 2.

In all explored blends, the increase in P(NDI20D-T2) content in the bi-component films leads to a decrease in $p$-type mobility and an increase in $n$-type mobility. The content in $\mathrm{P}(\mathrm{NDI} 20 \mathrm{D}-\mathrm{T} 2)$ is therefore an effective remote control for tuning the two mobilities, enabling the development of transistors with balanced mobilities for each blend. While these balanced mobilities were observed at $20 \mathrm{wt} \%$ of P(NDI2ODT2) blended with P3HT and PCD-TPT, the ratio for balanced mobilities PDVT-8 and IIDDT-C3 based transistors was found to be around $50 \mathrm{wt} \%$ of P(NDI2OD-T2).

The $I_{o n} / I_{\text {off }}$ of the balanced ambipolar devices based on blends was determined from the transfer curves (Fig. 3). Ambipolar devices using a blend composed of $80 \mathrm{wt} \%$ of P3HT featured mobilities below $10^{-3} \mathrm{~cm}^{2} / \mathrm{Vs}$ and $\mathrm{I}_{\text {on }} / \mathrm{I}_{\text {off }}$ below $10^{3}$ in saturation regime. Furthermore, two orders of magnitude drop in $p$-type mobility are observed upon addition of 20 wt $\%$ P(NDI20D-T2), combined with a steep increase in $n$-type charge transport. Such effect can be ascribed to hole-electron charge recombination that occurs when the n-type polymer, i.e. P(NDI2OD-T2), is mixed with the p-type polymer, i.e. P3HT. The well blended nature of the film is confirmed by Ambient-UPS measurements (Fig. S6a).
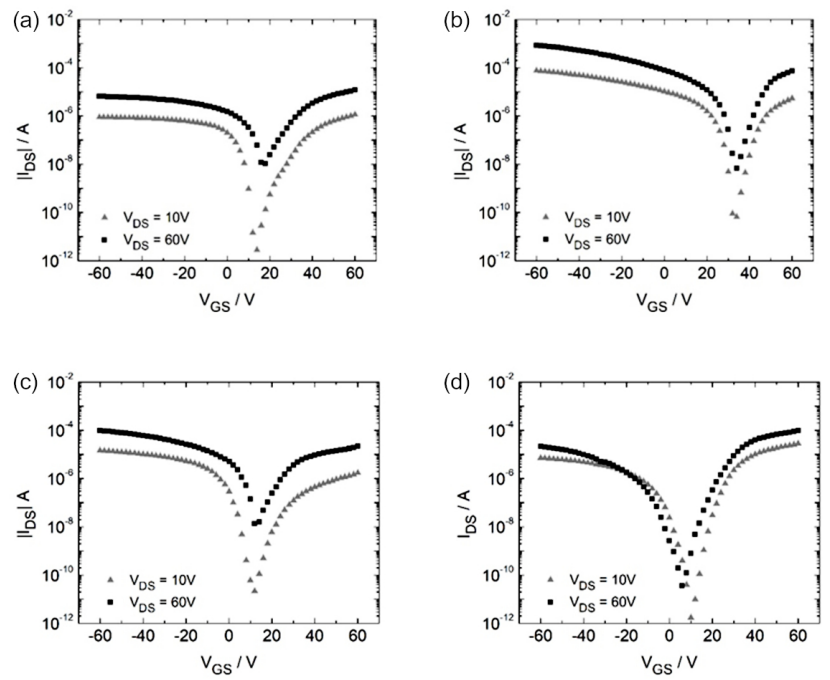

Figure 3. Transfer curves of transistors $(L=20 \mu \mathrm{m})$ produced from blends of $\mathrm{P}(\mathrm{NDI} 2 \mathrm{OD}-\mathrm{T} 2)$ and $p$-type semiconductors: $\begin{array}{lll}\text { (a) } \quad 80: 20 & \text { P3HT:P(NDI2OD-T2), (b) 80:20 PCD- }\end{array}$ TPT:P(NDI2OD-T2), (c) 50:50 PDVT-8:P(NDI2OD-T2), and (d) 50:50 IIDDT-C3:P(NDI2OD-T2). The scales on the voltage and current axes are the same in all graphs for the sake of comparison. In all cases, the gate voltage was swept from $-60 \mathrm{~V}$ to $+60 \mathrm{~V}$

PCD-TPT featured balanced mobilities when blended with 20 wt\% P(NDI20D-T2), with high electrical performances: the fabricated ambipolar transistors displayed balanced mobilities $>10^{-2} \mathrm{~cm}^{2} / \mathrm{Vs}\left(\mu_{\mathrm{p}}=0.015 \mathrm{~cm}^{2} / \mathrm{Vs} \approx \mu_{\mathrm{n}}=\right.$ $0.017 \mathrm{~cm}^{2} / \mathrm{Vs}$ ) and $\mathrm{I}_{\text {on }} / \mathrm{I}_{\text {off }}$ exceeding $10^{4}$ both in linear and saturation regime. These performances can be ascribed to the relatively high $p$ - and $n$-type mobility of PCD-TPT, and provide unambiguous evidence that this approach can be used to "correct" ambipolar polymers featuring unbalanced mobilities. IIDDT-C3 and PDVT-8 devices showed ambipolar transport with balanced mobilities when blended in equivalent weight quantities with P(NDI20D-T2). The extracted mobilities were slightly below $10^{-2} \mathrm{~cm}^{2} / \mathrm{Vs}$. In the case of 50:50 IIDDT-C3:P(NDI2OD-T2) devices, $\mathrm{I}_{\text {on }} / \mathrm{I}_{\text {off }}$ ratios exceeded $10^{5}$ in saturation regime and reached $10^{7}$ in linear regime, being the highest reported values to date for ambipolar organic field-effect transistors. Such a large high $\mathrm{I}_{\text {on }} / \mathrm{I}_{\text {off }}$ stems from a threshold voltage $\left(\mathrm{V}_{\mathrm{Th}}\right)$ "window" $\left(\Delta \mathrm{V}_{\mathrm{Th}}\right.$ $=\mathrm{V}_{\mathrm{Th}, \mathrm{n}}-\mathrm{V}_{\mathrm{Th}, \mathrm{p}}$ ) of $30 \mathrm{~V}$, where the device is off (Supporting Table 2).

Atomic Force Microscopy and Grazing Incidence Wide Angle X-ray Scattering (GIWAXS) measurements were carried out on polymer blends with 50:50 ratio in weight and compared to single-component films to investigate the effect of blending on the film's morphology (Fig. 4, Supporting Table 4, Fig. 5). Additional line profile analysis of GIWAXS images and XRR curves are reported in the Supporting Information, section 6. Moreover, Ambient Ultraviolet Photoelectron Spectroscopy (A-UPS) measurements were performed (Fig. S6) to gain greater insight into the possible occurrence of phase segregation along the Z-axis, as A-UPS is only able to probe the very first few nanometers on the top of the sample. All values of the measured Ionization Energy (IE) of the polymeric semiconductors and work function of the bare electrodes are reported in Supporting Table 5.

Films made from P3HT and P(NDI2OD-T2) blends did not display the ordered and parallel lamellae typical for pristine P(NDI2OD-T2) films in the AFM images, but instead larger aggregates of short and disordered structures yielding an increased roughness compared to both starting materials. This could be due to either P3HT aggregating into larger domains and mostly covering P(NDI2OD-T2), or to P(NDI2ODT2) losing its typical morphological features once blended with P3HT, or to both effects. The IE measured by A-UPS (Fig. S6a) was found to lie between those of P3HT and $\mathrm{P}(\mathrm{NDI} 2 \mathrm{OD}-\mathrm{T} 2)$, being a clear indication that on the top surface both components are present.

GIWAXS measurements performed on the P3HT:P(NDI2OD-T2) 50:50 blends confirmed a lower degree of crystalline order for both polymer aggregates (Fig. $\mathbf{5 a}, \mathbf{b}, \mathbf{f})$. The intensity of the peaks coming from P3HT and P(NDI2OD-T2) crystalline domains, normalized by the thickness (Fig. S18, supporting table 6), was lower than the ones observed in single-component films. Moreover, the typical high ordered edge-on configuration of pristine P3HT (Fig. 5b, S9) at the interface with the dielectric surface 32 disappeared in favor of the face-on configuration, where lamellas stack along the in-plane direction and the $\pi-\pi$ interaction lies perpendicularly to the film plane. This orientation could explain the reduction of two orders of magnitude in p-type mobility. GIWAXS patterns were recorded at different incident angles (Fig. S10), probing the film at different depths, from the first $\sim 9 \mathrm{~nm}$ close to the top surface to the whole film thickness. The intensity of all peaks gradually increased with the incident angle, revealing that the crystal domains of P3HT and P(NDI2OD-T2) were homogenously spread across the film. 
AFM images of PCD-TPT:P(NDI2OD-T2) 50:50 blends revealed a surface morphology similar to that of PCD-TPT, as quantified by a $R_{R M S}=0.8 \mathrm{~nm}$ in both cases. The close similarity between P(NDI2O D-T2) and PCD-TPT single-component films prevented determination of the surface composition by AFM. However, A-UPS (Fig. S6b) unambiguously indicated that the top surface is composed mostly of PCDTPT, since the HOMO value is identical between the blend and the pristine PCD-TPT film.

Both PCD-TPT and P(NDI2OD-T2) polymers generally tend to aggregate in low crystalline films and their blending did not strongly affect their structural features, as revealed by GIWAXS images (Fig. 5 a,c,g). All peaks broadened due to the decrease of crystalline domain size (Fig. S11). Strong variations were observed when the blend film was probed at different depths. Intensity of P(NDI2OD-T2) lamellar peaks observed in the in-plane direction (Fig. S12) strongly increased when the whole film was probed, whereas the reflection coming from the $\pi-\pi$ stacking of PCD-TPT remained almost unchanged. When the first $9 \mathrm{~nm}$ of the film were probed, the profiles of the integrated intensity along the out-of-plane and in-plane directions were close to the one obtained for pure PCD-TPT film (Fig. S13). These features indicate that the film surface is mostly covered by PCD-TPT polymer, confirming the conclusions drawn through A-UPS measurements. These segregation phenomena could explain the unchanged high n-type mobility measured for 20:80, 50:50, 80:20 and 0:100 blends, since the dielectric surface is mostly covered by PCD-TPT polymer bottom gate charged.

Regarding PDVT-8:P(NDI2OD-T2) 50:50 blends, the surface was rougher $\left(\Delta \mathrm{R}_{\mathrm{RMS}}=0.28 \mathrm{~nm}\right)$ than in the case of neat PDVT-8, but the surface morphology would exhibit very similar features to those measured in pristine films. Therefore, it is very likely that PDVT-8 is sitting on the top of the film, as confirmed by A-UPS (Fig S6c). However, the semiconductor-dielectric interface most certainly does not consist only of P(NDI2OD-T2), since blended films at such ratio exhibit balanced electron-hole mobilities. Furthermore, the presence of $20 \mathrm{wt} \% \mathrm{P}(\mathrm{NDI} 2 \mathrm{OD}-\mathrm{T} 2)$ within the blend did not lead to a notable modification of the electrical performances of the device, definitively ruling out the presence of a pure P(NDI20D-T2) layer at the interface.

GIWAXS measurements revealed that polymer organization strongly changed when PDVT-8 was blended with $\mathrm{P}$ (NDI2OD-T2) (Fig. 5d,h). From pure edge-on configuration observed in neat film, PDVT-8 polymer aggregated in blended film with both face-on and edge-on orientations and with lower crystalline order. All the Bragg peaks collected by probing $\sim 17 \mathrm{~nm}$ of film thickness did not strongly increase when the whole film was probed (Fig. S15), indicating the presence of amorphous material at the interface with the dielectric surface. Otherwise, peaks were strongly reduced, and bumps persisted when only the first $9 \mathrm{~nm}$ were probed, suggesting the presence of disordered material at the film surface as well. Since GIWAXS detects only the film crystallinity, the nature of the disordered material at the interface with the dielectric as well as at the film surface cannot be identified.
Since neither AFM, A-UPS nor GIWAXS measurements provided unambiguous information on the film composition, top-gate devices of PDVT-8, P(NDI2OD-T2) and 50:50 blends were fabricated and characterized to determine if the top surface of the films favored hole or electron transport. While the top surface of the sample might be affected by the deposition and annealing of the dielectric, a mild approach (annealing at $70^{\circ} \mathrm{C}$ was selected to avoid phase segregation as much as possible. Electrical characterization revealed that $p$-type mobility decreases by one order of magnitude in the case of PDVT-8 transistors in top-gate as compared to bottom-gate configuration. On the contrary, $\mathrm{P}(\mathrm{NDI} 2 \mathrm{OD}-\mathrm{T} 2)$ transistors in top-gate configuration featured mobilities one order of magnitude higher, as previously reported in the literature..$^{33-34}$ In general, such change in charge carrier mobility can be ascribed to the different electrode-semiconductor and dielectric-semiconductor interfaces. While P(NDI2OD-T2) benefitted from the increased injection area in the top-gate bottom-contact configuration (as was anticipated considering the injection barriers due to energy level differences), PDVT-8 devices with OTS-treated dielectric (in bottom-contact transistors) displayed higher mobilities, while not exhibiting much injection difficulties from the output curves reported in Fig. S2. Ambipolar transistors in top-gate configuration were fabricated and characterized (Fig. S7, S8). Films produced from blends of PDVT-8 and P(NDI2OD-T2) lead to a sharp decrease in $n$-type mobility (below $10^{-4} \mathrm{~cm}^{2} / \mathrm{Vs}$ ), while maintaining very high $p$-type mobility over $10^{-2} \mathrm{~cm}^{2} / V s$. Therefore, the electrical measurements, A-UPS data and the AFM analysis all together indicated that the top surface of that film is mostly composed of disordered PDVT-8.

Finally, films made from 50:50 IIDDT-C3:P(NDI2OD-T2) blended solutions featured the most interesting surface in AFM images. Unlike the other $p$-type polymers that, upon blending, displayed higher or similar roughness than in pristine films, the film surface exhibited reduced roughness when IIDDT-C3 was blended with P(NDI2OD-T2) (lowering from 1.78 to $0.85 \mathrm{~nm}$ ). This could be the results of smaller aggregates for each polymer, with a similar blend at the dielectric interface, explaining the strong effect of the presence of $20 \%$ of each polymer with the other on the electrical performances. A-UPS measurements seem to hint at a surface that is mostly, but not exclusively, covered with IIDDTC3 (Fig. S6d). The structural characterization of the film by means of GIWAXS (Fig 5i) did not reveal any segregation phenomena induced by the co-presence of two polymers during the self-assembly of the blend. Crystal domains of both the polymers are homogenously spread across the films made from 50:50 IIDDT-C3:P(NDI2OD-T2) blended solutions. Top-gate transistors using IIDDT-C3 and 50:50 IIDDT-C3: P(NDI2OD-T2) semiconductor solutions were fabricated and characterized (Fig. S7, S8) to further investigate any vertical phase segregation. Transistors fabricated using blends of IIDDT-C3 and P(NDI2OD-T2) at the 50:50 ratio, featured balanced mobilities exceeding $10^{-2}$ $\mathrm{cm}^{2} / \mathrm{Vs}$ in both cases. These results can be explained by the well-blended nature of the film, in accordance with the GIWAXS characterization. 


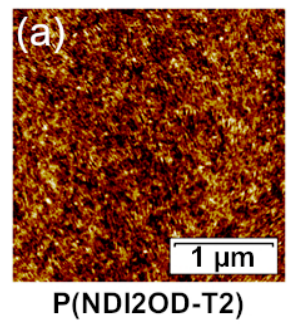

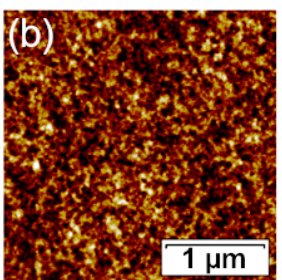

P3HT

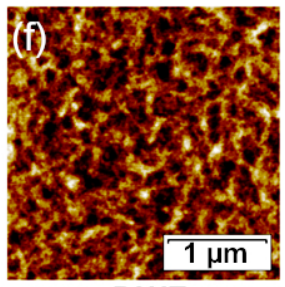

P3HT

$+\mathrm{P}(\mathrm{NDI} 2 \mathrm{OD}-\mathrm{T} 2)$

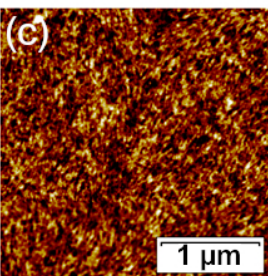

PCD-TPT

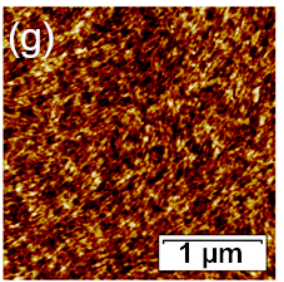

PCD-TPT

$+\mathrm{P}$ (NDI2OD-T2)

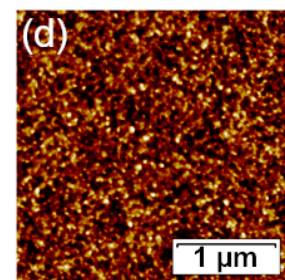

PDVT-8

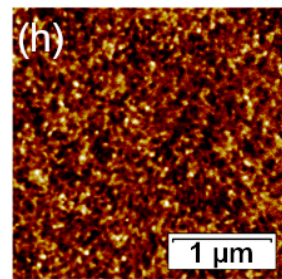

PDVT-8

$+\mathrm{P}$ (NDI2OD-T2)

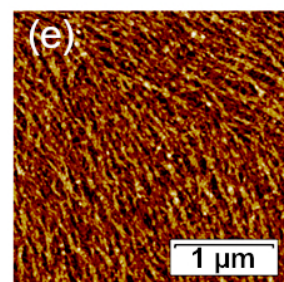

IIDDT-C3

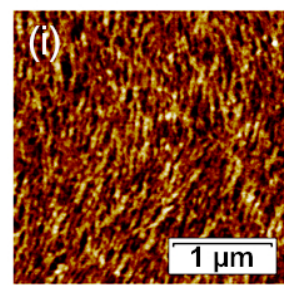

IIDDT-C3

$+\mathrm{P}(\mathrm{NDI} 2 \mathrm{OD}-\mathrm{T} 2)$

Figure 4. Tapping Mode AFM topography images of 50:50 blended polymer films. Z-scales: (a) $4.4 \mathrm{~nm}$, (b) $3.5 \mathrm{~nm}$, (c) $5.5 \mathrm{~nm}$, (d) $6.4 \mathrm{~nm}$, (e) $12.1 \mathrm{~nm}$, (f) $5.7 \mathrm{~nm}$, (g) $5.8 \mathrm{~nm}$, (h) $5.4 \mathrm{~nm}$, and (i) $5.8 \mathrm{~nm}$.
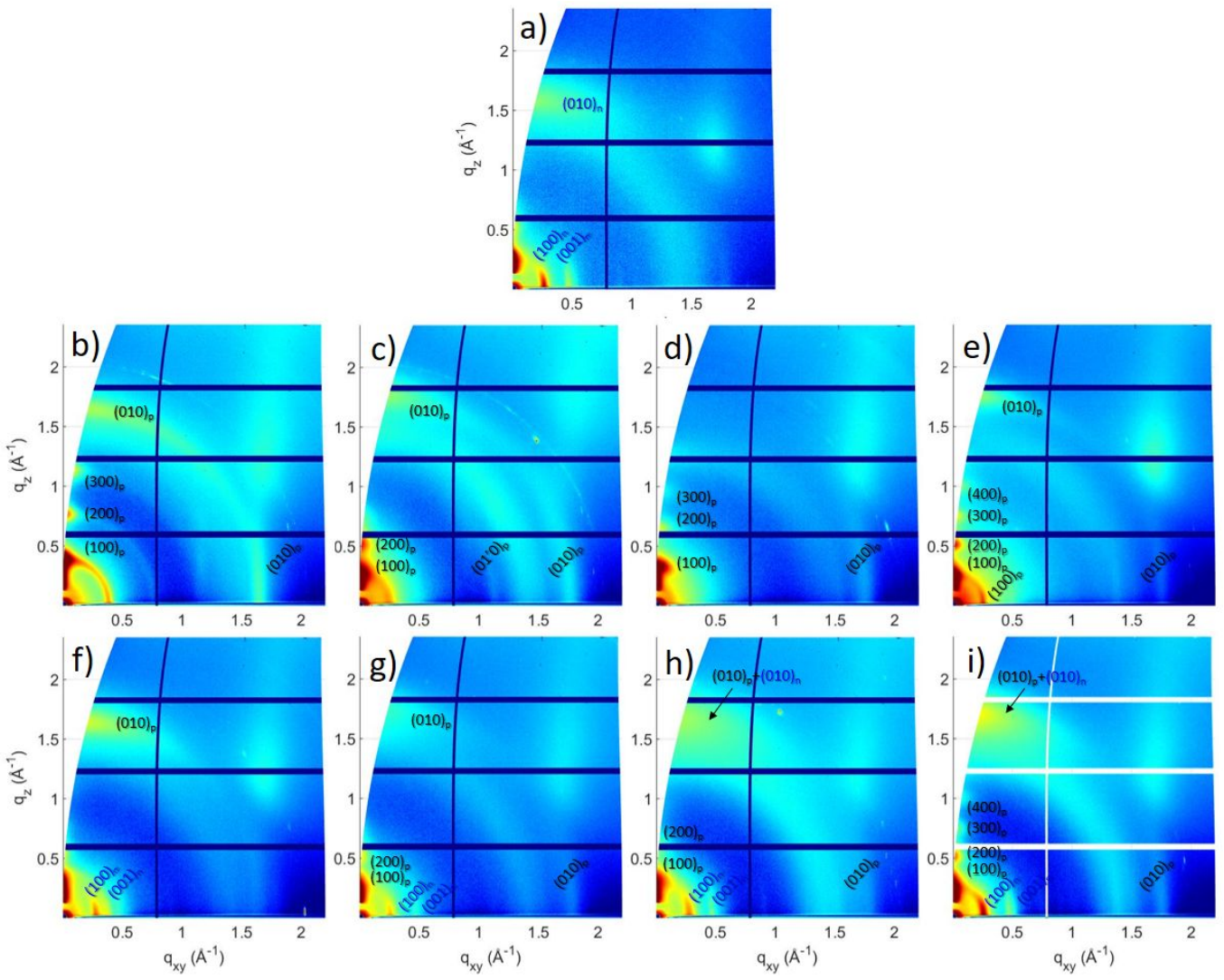

Figure 5. 2D-GIWAXS measurements. Images collected from single component films: (a) P(NDI2OD-T2), (b) P3HT, (c) PCDTPT, (d) PCVT-8 and (e) IIDDT-C3 and bi-component films: (f) P3HT:P(NDI2OD-T2), (g) PCD-TPT:P(NDI2OD-T2), (h) PCVT8:P(NDI2OD-T2), and (i) IIDDT-C3:P(NDI2OD-T2). 
Complementary-like inverter circuits were fabricated through the integration of two identical transistors produced from the three best performing polymer blends. This approach has so far been lacking in terms of performances, with very low gains and noise margins compared to complementary-like inverter circuits relying on two different transistors (one $p$-type, the other $n$-type). ${ }^{35-36}$ However, the use of an ambipolar solution reduces processing complexity, as a single semiconducting film can be deposited for all the transistors in the circuit (Fig. 6a). ${ }^{37}$ Complementary-like inverters based on blends of P(NDI20D-T2) with PCD-TPT and IIDDT-C3 were fabricated and tested. A gain of ca. 60 and Noise Margins (NM) over $30 \%$ of $1 / 2 V_{D D}$ were measured at $\mathrm{V}_{\mathrm{DD}}=30 \mathrm{~V}$ (Fig. 6b) for PCD-TPT based inverters. These high performances can be ascribed to the high $\mathrm{I}_{\text {on }} / \mathrm{I}_{\text {off }}$ and balanced mobilities. IIDDT-C3:P(NDI20D-T2) inverters featured lower performances (gain of 30 ) at $\mathrm{V}_{\mathrm{DD}}=30 \mathrm{~V}$, and negative noise margins due to the low off voltage $\left(\mathrm{V}\right.$ at $\mathrm{I}_{\mathrm{DS}}=$ $\left.I_{\text {min }}\right)$ of the transistors $\left(I_{\text {off,min }}\right.$ at $\left.V_{\mathrm{IN}}=5 \mathrm{~V}\right)$.
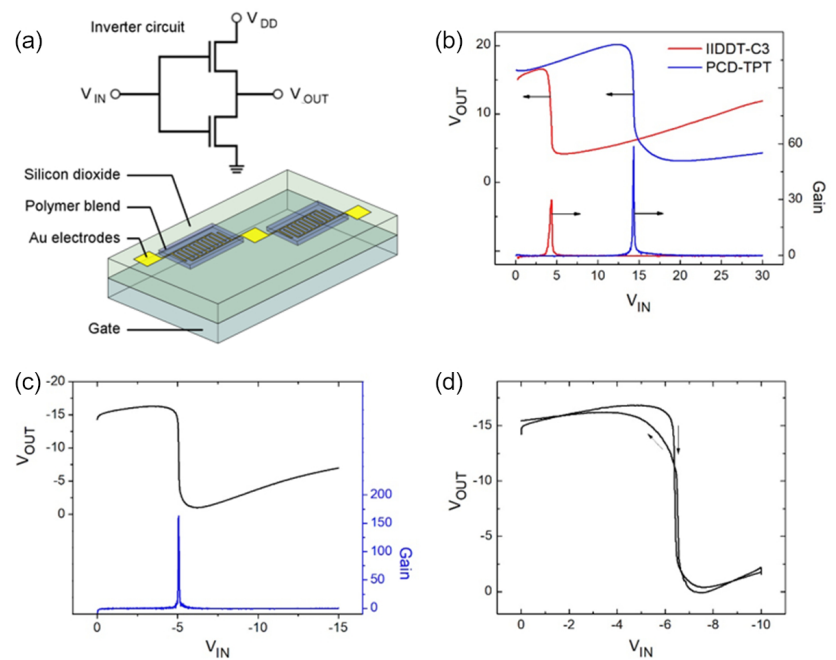

Figure 6. Inverter circuits based on ambipolar polymer blends. (a) Configuration of the inverters, made of transistors with $\mathrm{W}=10 \mathrm{~mm}$ and $\mathrm{L}=60 \mu \mathrm{m}$. The dielectric consisted of a $90 \mathrm{~nm}$ thick layer of $\mathrm{SiO}_{2}$. (b) Gain (left axis) and $\mathrm{V}_{\text {OUt }}$ (right axis) as a function of $V_{\text {IN }}$. Results obtained on an inverter fabricated using $\mathrm{P}$ (NDI2OD-T2) blended with PCD-TPT at 20:80 (blue lines) or IIDDT-C3 at 50:50 (red lines). $V_{D D}=30 \mathrm{~V}$. (c) Gain (left axis) and $V_{\text {OUT }}$ (right axis) as a function of $V_{\text {IN }}$. Results obtained on an inverter fabricated using P(NDI2OD-T2) blended with PDVT-8 at 50:50. $\mathrm{V}_{\mathrm{DD}}=-30 \mathrm{~V}$. (d) $\mathrm{V}_{\mathrm{OUT}}$ (right axis) as a function of $V_{\text {IN }}$. Results obtained on an inverter fabricated using $\mathrm{P}(\mathrm{NDI} 2 \mathrm{OD}-\mathrm{T} 2)$ blended with PDVT-8 at 50:50. $\mathrm{V}_{\mathrm{DD}}=30 \mathrm{~V}$.

Inverter circuits fabricated from 50:50 PNDI20DT2:PDVT-8 blends were characterized (Fig. 6c) and a very high gain value over 160 was measured, placing it among the state-of-the-art performances observed for ambipolar organic semiconductor-based inverters, ${ }^{38-39}$ using commercially available polymers and simple device fabrication. Previous studies have reported gains in the 10-40 range, even using semiconductors with high and balanced mobilities in the $0.1-10 \mathrm{~cm}^{2} /$ Vs range. ${ }^{40-43}$ However, in the case of our PNDI20D-T2:PDVT-8 inverters, balanced mobilities are complemented by a high $\mathrm{I}_{\text {on }} / \mathrm{I}_{\text {off }}$ and large $\Delta \mathrm{V}_{\text {Th }}$ over $10 \mathrm{~V}$ for both transistors (transfer and output curves reported in
Fig. S20 and Fig. S21). These parameters, in addition to the subthreshold swing in saturation regime determined to be comprised between $2 \mathrm{~V} / \mathrm{dec}$ and $3 \mathrm{~V} / \mathrm{dec}$ for both transistors, are of crucial importance in the case of an inverter circuit and explain the very high recorded gain. The noise margin amounted to $3.3 \mathrm{~V}(\mathrm{NM} \approx 20 \%$ of $1 / 2 \mathrm{VDD})$, being higher than inverters based on IIDDT-C3 containing solutions but below inverters based on PCD-TPT solutions (NM $>30 \%$ of $\left.1 / 2 V_{D D}\right)$. This result is consistent with the measured off-current of the PDVT-8:PNDI20D-T2 50:50 devices observed at $\mathrm{V}_{\text {IN }}=12 \mathrm{~V}$. The inverter circuits were tested at positive and negative $V_{\text {IN }}$ (Fig. S22) and in forward and reverse cycle (Fig. 6d); the inverters featured high gains over 60 in each case.

In summary, we have demonstrated that ambipolar organic transistors could be fabricated by using blends of $n$ type and $p$-type polymers. P(NDI20D-T2) was combined with four different $p$-type polymers, and each time it was possible to fabricate devices with balanced $p$ - and $n$-type mobilities and $\mathrm{I}_{\mathrm{on}} / \mathrm{I}_{\text {off }}$ over $10^{5}$ in linear regime. In particular, devices produced from P(NDI2OD-T2) blended with IIDDTC3 featured mobilities up to $10^{-2} \mathrm{~cm}^{2} / \mathrm{Vs}$ and $\mathrm{I}_{\mathrm{on}} / \mathrm{I}_{\text {off }}$ of $10^{7}$, being unprecedented values for ambipolar organic transistors. The morphology of the films was investigated by means of AFM and GIWAXS and findings were confirmed by electrical characterization performed using different transistor geometries (top-gate vs. bottom-gate), effectively probing the phase segregation by electrical testing. The fine control of the ratio between $p$ - and $n$-type mobility and state-of-the-art $\mathrm{I}_{\mathrm{on}} / \mathrm{I}_{\mathrm{off}}$ is particularly interesting for logic applications that rely on transistors with different polarities with good capabilities to switch off. This was highlighted by the fabrication of complementary-like inverter circuits through the integration of two identical ambipolar blend-based transistors, with 3 pairs of polymers. PCD-TPT and PDVT-8-based inverter circuits featured exceptionally high performances, with high voltage gains (over 60 and 160 respectively) and good noise margins (NM $>30 \%$ of $1 / 2$ $\mathrm{V}_{\mathrm{DD}}$ and $\mathrm{NM}>20 \%$ of $1 / 2 \mathrm{~V}_{\mathrm{DD}}$ respectively), demonstrating that our approach is effective for high-performance inverter fabrication. These state-of-the-art performances were furthermore achieved using a modest $\mathrm{V}_{\mathrm{DD}}$ of $-30 \mathrm{~V}$, highlighting the potential for device integration in real-life applications. The systematic study presented in this article allows the determination of important parameters when considering polymers for ambipolar blends. Materials composing the films used in high-performance inverter circuits should feature the characteristics of the two best performing polymers, PCD-TPT and PDVT-8. In particular, high electrical performances of each polymer (mobility, $\mathrm{I}_{\text {on }} / \mathrm{I}_{\text {off }}$ ) are a major requirement. Furthermore, both blends leading to well performing inverter circuits featured an amorphous phase, while films of P(NDI2OD-T) blended with P3HT or IIDDTC3 retained separate crystalline domains upon solvent evaporation. Most importantly, the approach described in this article is versatile and tunable: the reported results could be surpassed by selection of other higher-performing materials as soon as they will be commercially available.

\section{ASSOCIATED CONTENT}

Supporting Information. Experimental details and additional data including electrical characterization, AFM, A-UPS, GIWAXS 
are available in the supporting information. This material is available free of charge via the Internet at http://pubs.acs.org.

\section{Corresponding Author}

Paolo Samorì. E-mail: samori@unistra.fr. Emanuele Orgiu. E-mail: emanuele.orgiu@emt.inrs.ca.

\section{Present Addresses}

$\S$ Present address: INRS-Centre Énergie Matériaux Télécommunications, 1650 Blv. Lionel-Boulet, J3X 1S2 Varennes, Québec.

\section{Author Contributions}

The manuscript was written through contributions of all authors. All authors have given approval to the final version of the manuscript.

\section{Notes}

The authors declare no competing financial interests.

\section{ACKNOWLEDGMENT}

This work was financially supported by Région Grand Est project HARWEST, EC through the ERC project SUPRAFUNCTION (GA-257305) and Marie Sklodowska-Curie ETN projects iSwitch (GA No. 642196) and the SYNCHRONICS (GA 643238), the Labex project CSC (ANR-10- LABX-0026 CSC) within the Investissement d'Avenir program ANR-10-IDEX-0002-02, and the International Center for Frontier Research in Chemistry (icFRC).

\section{REFERENCES}

1. Pfleiderer, H.; Kusian, W., Ambipolar field-effect transistor. Solid State Electron. 1986, 29 (3), 317-319.

2. $\quad$ Chesterfield, R. J.; Newman, C. R.; Pappenfus, T. M.; Ewbank, P. C.; Haukaas, M. H.; Mann, K. R.; Miller, L. L.; Frisbie, C. D., High electron mobility and ambipolar transport in organic thinfilm transistors based on a pi-stacking quinoidal terthiophene. $A d v$. Mater. 2003, 15 (15), 1278-1282.

3. Zaumseil, J.; Sirringhaus, H., Electron and ambipolar transport in organic field-effect transistors. Chem. Rev. 2007, 107 (4), 1296-1323.

4. Bisri, S. Z.; Piliego, C.; Gao, J.; Loi, M. A., Outlook and Emerging Semiconducting Materials for Ambipolar Transistors. Adv. Mater. 2014, 26 (8), 1176-1199.

5. Chua, L. L.; Zaumseil, J.; Chang, J. F.; Ou, E. C. W.; Ho, P. K. H.; Sirringhaus, H.; Friend, R. H., General observation of n-type field-effect behaviour in organic semiconductors. Nature $\mathbf{2 0 0 5}$ 434 (7030), 194-199.

6. Zhou, K.; Dong, H. L.; Zhang, H. L.; Hu, W. P., High performance n-type and ambipolar small organic semiconductors for organic thin film transistors. Phys. Chem. Chem. Phys. 2014, 16 (41), 22448-22457.

7. Dou, L. T.; Liu, Y. S.; Hong, Z. R.; Li, G.; Yang, Y., LowBandgap Near-IR Conjugated Polymers/Molecules for Organic Electronics. Chem. Rev. 2015, 115 (23), 12633-12665.

8. Zhao, Y.; Guo, Y. L.; Liu, Y. Q., 25th Anniversary Article: Recent Advances in n-Type and Ambipolar Organic Field-Effect Transistors. Adv. Mater. 2013, 25 (38), 5372-5391.

9. Shi, K. L.; Zhang, W. F.; Gao, D.; Zhang, S. Y.; Lin, Z. Z.; Zou, Y.; Wang, L. P.; Yu, G., Well-Balanced Ambipolar Conjugated Polymers Featuring Mild Glass Transition Temperatures Toward HighPerformance Flexible Field-Effect Transistors. Adv. Mater. 2018 , $30(9), 1705286$.

10. Mas-Torrent, M.; Rovira, C., Novel small molecules for organic field-effect transistors: towards processability and high performance. Chem. Soc. Rev. 2008, 37 (4), 827-838.
11. Xue, G. B.; Fan, C. C.; Wu, J. K.; Liu, S.; Liu, Y. J.; Chen, H. Z.; Xin, H. L.; Li, H. Y., Ambipolar charge transport of TIPS-pentacene single-crystals grown from non-polar solvents. Mater. Horiz. 2015, 2 (3), 344-349.

12. Dodabalapur, A.; Katz, H. E.; Torsi, L.; Haddon, R. C., Organic field-effect bipolar transistors. Appl. Phys. Lett. 1996, 68 (8), 1108-1110.

13. Dinelli, F.; Capelli, R.; Loi, M. A.; Murgia, M.; Muccini, M.; Facchetti, A.; Marks, T. J., High-mobility ambipolar transport in organic light-emitting transistors. Adv. Mater. 2006, 18 (11), 1416 1420.

14. Rost, C.; Karg, S.; Riess, W.; Loi, M. A.; Murgia, M.; Muccini, M., Ambipolar light-emitting organic field-effect transistor. Appl. Phys. Lett. 2004, 85 (9), 1613-1615.

15. Cho, S.; Yuen, J.; Kim, J. Y.; Lee, K.; Heeger, A. J.; Lee, S. Multilayer bipolar field-effect transistors. Appl. Phys. Lett. 2008, 92 (6), 063505-063505-3.

16. Wei, Q. S.; Tajima, K.; Hashimoto, K., Bilayer Ambipolar Organic Thin-Film Transistors and Inverters Prepared by the Contact-Film-Transfer Method. ACS Appl. Mater. Inter. 2009, 1 (9), 1865-1868.

17. Kim, F. S.; Ahmed, E.; Subramaniyan, S.; Jenekhe, S. A., AirStable Ambipolar Field-Effect Transistors and Complementary Logic Circuits from Solution-Processed n/p Polymer Heterojunctions. ACS Appl. Mater. Inter. 2010, 2 (11), 2974-2977.

18. Kim, J.; Khim, D.; Yeo, J. S.; Kang, M.; Baeg, K. J.; Kim, D. Y., Polymeric P-N Heterointerface for Solution-Processed Integrated Organic Optoelectronic Systems. Adv. Opt. Mater. 2017, 5 (23), 1700655.

19. Shkunov, M.; Simms, R.; Heeney, M.; Tierney, S.; McCulloch, I., Ambipolar field-effect transistors based on solution-processable blends of thieno[2,3-b]thiophene terthiophene polymer and methanofullerenes. Adv. Mater. 2005, 17 (21), 2608.

$20 . \quad J a n a s z$, L.; Luczak, A.; Marszalek, T.; Dupont, B. G. R.; Jung, J.; Ulanski, J.; Pisula, W., Balanced Ambipolar Organic Field-Effect Transistors by Polymer Preaggregation. ACS Appl. Mater. Inter. 2017, 9 (24), 20696-20703.

21. Kang, M.; Hwang, H.; Park, W. T.; Khim, D.; Yeo, J. S.; Kim Y.; Kim, Y. J.; Noh, Y. Y.; Kim, D. Y., Ambipolar Small-Molecule:Polymer Blend Semiconductors for Solution-Processable Organic FieldEffect Transistors. ACS Appl. Mater. Inter. 2017, 9 (3), 2686-2692.

22. Earmme, T.; Hwang, Y. J.; Murari, N. M.; Subramaniyan, S.; Jenekhe, S. A., All-Polymer Solar Cells with 3.3\% Efficiency Based on Naphthalene Diimide-Selenophene Copolymer Acceptor. J. Am. Chem. Soc. 2013, 135 (40), 14960-14963.

23. Fabiano, S.; Himmelberger, S.; Drees, M.; Chen, Z. H.; Altamimi, R. M.; Salleo, A.; Loi, M. A.; Facchetti, A., Charge Transport Orthogonality in All-Polymer Blend Transistors, Diodes, and Solar Cells. Adv. Energy Mater. 2014, 4 (6), 1301409.

24. Szendrei, K.; Jarzab, D.; Chen, Z. H.; Facchetti, A.; Loi, M A., Ambipolar all-polymer bulk heterojunction field-effect transistors. J. Mater. Chem. 2010, 20 (7), 1317-1321.

25. Lu, C.; Wang, J.; Chang, H. C.; Chiu, Y. C.; Chen, H. Y.; Wu H. C.; Higashihara, T.; Chen, W. C., Ambipolar field-effect transistors using conjugated polymers with structures of bilayer, binary blends, and paralleled nanofibers. J. Mater. Chem. C 2014, 2 (36), 7489-7493.

26. Nam, S.; Seo, J.; Han, H.; Kim, H.; Bradley, D. D. C.; Kim, Y., Efficient Deep Red Light-Sensing All-Polymer Phototransistors with p-type/n-type Conjugated Polymer Bulk Heterojunction Layers. ACS Appl. Mater. Inter. 2017, 9 (17), 14983-14989.

27. Leclère, P.; Hennebicq, E.; Calderone, A.; Brocorens, P.; Grimsdale, A. C.; Müllen, K.; Brédas, J. L.; Lazzaroni, R., Supramolecular organization in block copolymers containing a conjugated segment: a joint AFM/molecular modeling study. Prog. Polym. Sci. 2003, 28 (1), 55-81.

28. Leclère, P.; Surin, M.; Jonkheijm, P.; Henze, O.; Schenning, A. P. H. J.; Biscarini, F.; Grimsdale, A. C.; Feast, W. J.; Meijer, E. W. Müllen, K.; Brédas, J. L.; Lazzaroni, R., Organic semi-conducting 
architectures for supramolecular electronics. Eur. Polym. J. 2004, 40 (5), 885-892.

29. Chen, Z. H.; Zheng, Y.; Yan, H.; Facchetti, A., Naphthalenedicarboximide- vs Perylenedicarboximide-Based Copolymers. Synthesis and Semiconducting Properties in Bottom-Gate N-Channel Organic Transistors. J. Am. Chem. Soc. 2009, 131 (1), 8-9.

30. Noriega, R.; Rivnay, J.; Vandewal, K.; Koch, F. P. V.; Stingelin, N.; Smith, P.; Toney, M. F.; Salleo, A., A general relationship between disorder, aggregation and charge transport in conjugated polymers. Nat. Mater. 2013, 12 (11), 1038-1044.

31. Luzio, A.; Fazzi, D.; Natali, D.; Giussani, E.; Baeg, K. J.; Chen, Z. H.; Noh, Y. Y.; Facchetti, A.; Caironi, M., Synthesis, Electronic Structure, and Charge Transport Characteristics of Naphthalenediimide-Based Co-Polymers with Different Oligothiophene Donor Units. Adv. Funct. Mater. 2014, 24 (8), 1151-1162.

32. Duong, D. T.; Toney, M. F.; Salleo, A., Role of confinement and aggregation in charge transport in semicrystalline polythiophene thin films. Phys. Rev. B 2012, 86 (20), 205205-205205-5.

33. Yan, H.; Chen, Z. H.; Zheng, Y.; Newman, C.; Quinn, J. R.; Dötz, F.; Kastler, M.; Facchetti, A., A high-mobility electron-transporting polymer for printed transistors. Nature 2009, 457 (7230), 679-686.

34. Schmidt, G. C.; Hoft, D.; Haase, K.; Hubler, A. C.; Karpov, E.; Tkachov, R.; Stamm, M.; Kiriy, A.; Haidu, F.; Zahn, D. R. T.; Yan, H.; Facchetti, A., Naphtalenediimide-based donor-acceptor copolymer prepared by chain-growth catalysttransfer polycondensation: evaluation of electrontransporting properties and application in printed polymer transistors. J. Mater. Chem. C 2014, 2 (26), 51495154.

35. Baeg, K. J.; Caironi, M.; Noh, Y. Y., Toward Printed Integrated Circuits based on Unipolar or Ambipolar Polymer Semiconductors. Adv. Mater. 2013, 25 (31), 4210-4244.
36. Li, H. Y.; Kim, F. S.; Ren, G. Q.; Jenekhe, S. A., High-Mobility n-Type Conjugated Polymers Based on Electron-Deficient Tetraazabenzodifluoranthene Diimide for Organic Electronics. J. Am. Chem. Soc. 2013, 135 (40), 14920-14923.

37. Cheng, S. S.; Huang, P. Y.; Ramesh, M.; Chang, H. C.; Chen, L. M.; Yeh, C. M.; Fung, C. L.; Wu, M. C.; Liu, C. C.; Kim, C.; Lin, H. C.; Chen, M. C.; Chu, C. W., Solution-Processed Small-Molecule Bulk Heterojunction Ambipolar Transistors. Adv. Funct. Mater. 2014, 24 (14), 2057-2063.

38. Nakano, M.; Osaka, I.; Takimiya, K., Control of Major Carriers in an Ambipolar Polymer Semiconductor by Self-Assembled Monolayers. Adv. Mater. 2017, 29 (1), 1602893.

39. Chen, Z. H.; Gao, D.; Huang, J. Y.; Mao, Z. P.; Zhang, W. F.; Yu, G., Thiazole-Flanked Diketopyrrolopyrrole Polymeric Semiconductors for Ambipolar Field-Effect Transistors with Balanced Carrier Mobilities. ACS Appl. Mater. Inter. 2016, 8 (50), 34725-34734.

40. Chen, Z. Y.; Lee, M. J.; Ashraf, R. S.; Gu, Y.; Albert-Seifried, S.; Nielsen, M. M.; Schroeder, B.; Anthopoulos, T. D.; Heeney, M.; McCulloch, I.; Sirringhaus, H., High-Performance Ambipolar Diketopyrrolopyrrole-Thieno[3,2-b]thiophene Copolymer FieldEffect Transistors with Balanced Hole and Electron Mobilities. Adv. Mater. 2012, 24 (5), 647-652.

41. Yoo, H.; Ghittorelli, M.; Lee, D. K.; Smits, E. C. P.; Gelinck, G. H.; Ahn, H.; Lee, H. K.; Torricelli, F.; Kim, J. J., Balancing Hole and Electron Conduction in Ambipolar Split-Gate Thin-Film Transistors. Sci. Rep. 2017, 7, 5015.

42. Kim, B.; Geier, M. L.; Hersam, M. C.; Dodabalapur, A., Inkjet printed circuits based on ambipolar and p-type carbon nanotube thin-film transistors. Sci. Rep. 2017, 7, 39627.

43. Lee, S. H.; Khim, D.; Xu, Y.; Kim, J.; Park, W. T.; Kim, D. Y.; Noh, Y. Y., Simultaneous Improvement of Hole and Electron Injection in Organic Field-effect Transistors by Conjugated Polymerwrapped Carbon Nanotube Interlayers. Sci. Rep. 2015, 5, 10407. 